\title{
Diversity in action: \\ Solutions for a more diverse and inclusive decade of planetary science and astrobiology
}

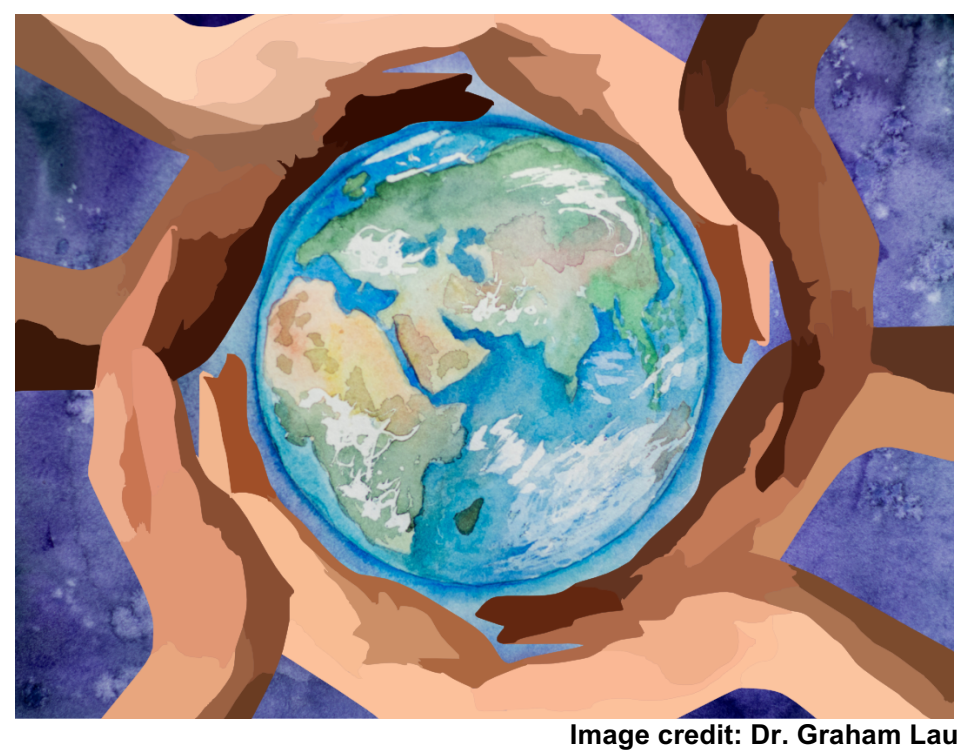

Authors:

Britney Schmidt (britneys@eas.gatech.edu), Oceans Across Space \& Time, Georgia Institute of Technology, Atlanta, GA

Sanjoy Som (sanjoy@bmsis.org), 206-775-8787, Oceans Across Space \& Time, Blue Marble Space Institute of Science, Seattle WA

Enrica Quartini (equartini@gatech.edu), Oceans Across Space \& Time, Georgia Institute of Technology, Atlanta, GA

Jacob Buffo, (jacob.j.buffo@dartmouth.edu), Oceans Across Space \& Time, Dartmouth College, Hanover, $\mathrm{NH}$

Chase Chivers (cchivers@gatech.edu), Georgia Institute of Technology, Atlanta, GA Krista Soderlund (krista@ig.utexas.edu), 218-349-3006, Oceans Across Space \& Time, The University of Texas at Austin, Institute for Geophysics, Austin, TX

Christopher E. Carr, (cecarr@gatech.edu), 617-216-5012, Oceans Across Space \& Time, Georgia Institute of Technology, Atlanta, GA

Benjamin Klempay, Oceans Across Space \& Time, Scripps Institution of Oceanography, UC San Diego, La Jolla, CA

Jeff Bowman (jsbowman@ucsd.edu), 425-753-3735, Oceans Across Space \& Time, Scripps Institution of Oceanography, UC San Diego, La Jolla, CA

To View the 113+ Endorsements (as of 09/14/2020), please follow this link: https://tinyurl.com/DiversityInAction 


\section{Motivation}

The Planetary Sciences and Astrobiology (PS\&A) communities have historically been challenged by low racial and ethnic diversity, especially with regard to Black scientists. A recent demographics survey of our community titled "Survey of Planetary Scientists" [1a] demonstrates a dominantly white community. While the US population is $13 \%$ Black, preliminary results of the survey indicate that the PS\&A community is $\sim 1.5 \%$ Black $[1 \mathrm{~b}, 1 \mathrm{c}]$. Dismantling systemic racism is not only a moral and ethical imperative. More diverse teams are better performing teams [2]. A more diverse PS\&A community can thus only benefit the community's progress towards realizing NASA objectives.

While true cultural change may be generational, there are short-term changes to NASA programs, institutional structures, and incentives that have the potential to markedly improve the status quo. For the first time, the Planetary Science and Astrobiology Decadal Survey will include workforce issues explicitly, which we believe is the first step in addressing necessary long term changes.

At the same time, COVID-19 and recent acts of police violence against Black people have undoubtedly affected communities differently. In particular, Black communities have been more severely affected than white communities, and women have been more severely affected than men, which may have an unintended negative impact on the range of ideas and missions submitted by the community for this decadal survey $[3,4]$. We thus encourage the decadal survey panel to poll the diversity of the White Paper submitters to formally understand which communities were not able to voice their opinion on the direction of our field for the next decade, and work to remove bias from the decadal panels through training, including under-represented individuals as panel members and presenters, and keeping lines of communication open with the community. The consequences of inadvertently alienating entire communities from the process may extend beyond the decade.

\section{Suggestions for increasing the diversity of the PS\&A community:}

\section{Diversity and Equity Training for Funded Investigators and Teams}

While most universities require Pls and graduate students to be trained in the ethical conduct of research in order to meet NSF grant requirements, NASA has comparatively few institutional requirements with respect to conduct of research, which includes practices that are inclusive. Many individual institutions provide optional diversity and equity training for their employees, yet this is not universally the case. A standard practice for a number of funding organizations (e.g., NSF) is the requirement of comparable training for its grantees to ensure a fundamental level of education and awareness around these important topics, and include such considerations as criteria for evaluating grants, such as NSF's Broader Impacts.

In planetary science, a discipline where both the general demographic and leadership roles are disproportionately dominated by one group and reports of harassment and concerns for safety are most prevalent amongst minority groups [7], formal and mandatory training is imperative in moving towards a safe, inclusive, and diverse environment for everyone in the community. Such interventional education and training has been shown to break habitual bias and improve the academic climate 
in regards to equity and advancement opportunity for under-represented groups [8]. If the institutions that support funded Pls and Co-ls do not offer such training, a reimbursement program for attending professional development on diversity-relevant topics could bridge this gap.

Recommendation: NASA, as the principal funding source for PS\&A investigations, should require diversity training for funded investigators and their teams on regular intervals, because best practices change with time. NASA Planetary Sciences \& Astrobiology programs should also consider how to implement administrator's Bridenstine's policy statement and incorporate inclusivity in the submission and review of grant proposals.

\section{$\underline{\text { 2. Faculty and Staff Fellowship Programs for Minorities at Non-R1 Institutions. }}$}

The NASA Early Career Fellowship (ECF) \& Early Career Award (ECA) are programs that were intended originally to increase the number of planetary science faculty at universities, in order to train more planetary scientists. Currently, the ECF program is only available to the PI or Science PI of a funded award. We suggest that the NASA ECF \& ECA program should be extended to funded early-career minority Co-ls as well in order to help them achieve permanent positions.

Another program could also increase the number of professors working in non-R1 programs that serve a broader swath of the United States, including Historically Black Colleges and Universities (HBCUs), Native American/Tribal Schools, Minority-serving Institutions and Liberal Arts Colleges, and other Minority Serving Institutions (henceforth MSIs). While the NASA ECA program has been envisioned as an increase in resources to help early career scientists along the path to long-term research-based careers, a similar program that is not tied to being awarded a PI grant could help increase the number of PS\&A faculty members who interact with minority students for whom non-R1 programs are more appealing. Non-R1 institutions often do not place the same value on securing extramural funding as other institutions, which can lead to MSI faculty choosing not to propose or lead grants. Lessons learned from the dominantly-R1 Minority Institution Research Sabbatical (MIRS) program (2002-2017) have signaled the need to expand the eligibility of such a program.

At the same time, those institutions value and incentivize service and have ongoing programs for diversity and inclusion, so increasing the presence of PS\&A in these institutions could help make our field more approachable to minority groups. We, therefore, suggest a NASA Diversity Fellows program to facilitate NASA-funded scientists to engage with faculty at institutions that are less motivated by grant success as a metric for faculty hiring and promotion.

Recommendation: NASA should study ways to implement programs like the NIH's Pathway to Independence Program for postdocs, and invite the broader community to contribute programmatic ideas via solicitations that enhance the number of PS\&A faculty at MSIs. Programs that connect with faculty and staff at non-R1 institutions similar in nature to the successful NASA ECF program, and build on lessons learned from the MIRS program would also proactively improve ED\&I. 


\section{Extended NASA Partnerships with MSIs}

Faculty and staff at MSIs are poorly represented in the community of PIs and Co-ls that compose PS\&A. Yet, these faculty train the next generation of scientists that could help address existing workforce imbalances that this decadal survey process seeks to address. The NASA/NSF Minority University Research and Education Project (MUREP) program represents an excellent step in the process for MSIs to receive funding and provide NASA experiences for minority students. However, direct connection with NASA-funded research investigators remains elusive. NASA could consider ways to promote research collaboration with MSIs. Such collaborations could receive additional consideration in the allocation of funding. The expansion of the NASA Pathways program may also provide direct connections between under-represented students and NASA jobs. Existing programs (such as this one and this one) provide potential models for expanded efforts.

A formal research partnership program between NASA and MSIs, overseen by a dedicated Program Officer (See Recommendation 9) that involves students directly conducting NASA research would provide clearer pathways and career options for students of these institutions. A dedicated research grant program for science faculty and staff at minority serving institutions could also help increase the number of Pls of diverse backgrounds.

Recommendation: NASA should develop and enhance existing programs that enable more active participation of PIs, Co-ls and students from MSIs in NASA research to help present planetary sciences and astrobiology as viable career options for students.

\section{Student Fellowships for Minorities}

A hurdle for many minorities is achieving admission and obtaining financial resources to support their education and research. Graduate fellowship programs such as FINESST (NASA) and the GRFP (NSF) are awarded disproportionately to students at highly ranked, R1 research institutions, even when directed towards minorities (e.g., GRFP and the discontinued Harriett G. Jenkins Predoctoral Fellowship). To achieve diversity and equity in the PS\&A community, investment must be made in supporting and retaining early career scientists whose primary scientific objectives may not be R1 research. Such an initiative could complement educational/research partnerships between R1 and minority serving institutions, and should be structured and communicated differently from FINESST.

Recommendation: We recommend that NASA revive and expand The Harriett G. Jenkins Pre-doctoral Fellowship Project (JPFP), which supported students from underrepresented communities, students with disabilities and low income/first generation students. In addition to graduate stipends and summer research opportunities, the award provided a community of awardees that would meet and share their research annually. Today, there is still an active JPFP alumni network. Meeting fellow students provides a network for advice and support, similar to faculty cluster hiring practices. 


\section{Establish a Mechanism to Facilitate Teacher-Scientist Collaborations in Science Education}

With movement away from public engagement components of proposals, there are limited resources available for a proposal team to prioritize and organize community engagement/education activities, yet those activities are immensely valuable. Identifying venues and opportunities for inspiring and educating a diverse group of young people is a priority of both NASA and proposal team scientists - the future generations of researchers. While centralized committees and funding for STEM education exist within NASA, there is limited interaction between PIs/Co-Is/Researchers of funded grants and $\mathrm{K}-12$ students, particularly in communities that have not historically been engaged with.

Recommendation: We suggest that NASA facilitates a program that pairs interested grantees and project teams with $\mathrm{K}-12$ educators and institutions in untapped communities to increase and broaden public engagement efforts.

\section{$\underline{6 .}$ Public Engagement Component on ROSES Grants}

Few federal agencies can inspire the next generation the way NASA can. Similarly, Few individuals can inspire the next generation in the same way that NASA PIs, Co-Is, and Collaborators can. Yet, community engagement activities have been removed from ROSES solicitations. As a tax-payer funded agency, NASA's grantees have the purview of sharing the knowledge gained with such funding, including the experience of science, to the next generation. Science outreach activities benefit all those involved.

The advantages are well understood. Science outreach programs to K-12 students may improve confidence in the classroom and future pursuit of careers in science [5]. Furthermore, scientists involved in public engagement benefit from gaining skills in communication and a deeper understanding of diversity and education issues [6]. Targeting public engagement programs for minorities in planetary science and astrobiology can not only serve as a type of diversity education, but also foster an interest in the PS\&A community for under-represented groups.

Recommendation: We recommend returning both optional and required public engagement and education objectives to ROSES solicitations (including competed missions) and enabling selected PIs to inspire future generations of scientists. We also recommend that NASA evaluate the efficacy of centralizing education, public outreach, and community engagement activities over the past decade.

\section{Revival of the Astrobiology Summer/Winter Schools \& Expansion of Training}

For almost a decade under the combined leadership of the NASA Astrobiology Institute and the Nordic Network for Astrobiology, yearly summer and winter schools were held in Iceland and Hawaii, respectively. These schools were fundamental to connecting earlycareer researchers with their peers from different institutions and creating lifelong connections that still bear fruit today. This community is also more diverse along many metrics than the broader PS\&A community. Reviving these schools, and forming new ones with an added emphasis on recruiting from untapped communities, will create a 
culturally and ethnically diverse early-career collaborative community whose impact will be career-long. Other critical early career serving activities that are student-led such as AbGradCon and LunGradCon should be given increased funding and assistance for including members of MSls.

Recommendation: We recommend re-starting and expanding schools for early-career PS\&A scientists beyond current themes of astrobiology and the Moon, with an added focus on recruiting minority students.

\section{Increase Value of Service Activities}

Community engagement must be further recognized and valued as an activity performed by PS\&A community members. While a public properly informed on scientific findings can make demands to their representatives, NASA findings can impact national policies directly as well. Value in community engagement activities by scientists have improved since the days of Carl Sagan. Yet, they still play a minor role in determining academic promotion and other career success metrics. Many minority faculty are particularly passionate about returning knowledge to and empowering their communities (and, as they are few, they also tend to receive many more requests from students as well as committees), which can take time away from writing publications and seeking grants. In today's climate, valorizing grants received over service can negatively impact the perceived value of community-serving efforts. Showcasing the efforts of scientists who are successful in both service and science will increase the profile of these individuals, rewarding their contribution and helping incentivize service. Consider how resources can be distributed to service-minded teams and individuals. The NSF CAREER program is a potential model for how to incentivize and reward coupled scientific innovation and effective service.

Recommendation: We recommend establishing a platform to further promote the values of service activities to continue to empower scientists to engage their communities and representatives, that includes messaging and action.

\section{Establish a Program Officer Dedicated to Diversity, Equity, and Inclusion}

Many of the suggestions offered above necessitate a sustained effort and dedicated personnel, and must outlive the dedication of a few passionate individuals. A culture of diversity, equity, and inclusion must visibly exist at NASA to lead change at NASAfunded institutions.

And yet, a culture fully accepting of minorities and aware of their unique challenges will lag any implementation of policies to improve the status quo. This may maintain alienating atmospheres in environments that have responded positively to change. Such atmospheres may not be conducive to the continued progression of individuals within PS\&A. Representation of minorities in leadership positions promotes voices that can better recognize, address, and provide support for the unique challenges facing these communities. Currently, the informal MIAC initiative (Minority Institute Astrobiology Collaborative) is one of the only venues where minorities can share experiences. 
Recommendation: We recommend establishing a new Program Officer within NASA and/or PS\&A focused on diversity, equity, and inclusion. This Officer's role, and their office, would be to foster community with, and provide support to, individuals to minimize a brain drain of under-represented minorities in advanced scientific pursuits relevant to planetary sciences and astrobiology. This new office could also help evaluate the effectiveness of ED\&I programs and procedural changes and serve as a repository of best practices and lessons learned.

\section{Updating NASA's Lanquage}

NASA's language must broaden its target audience. Representing NASA's culture in terms of valuing interdependence norms (i.e. being part of a community, most valued in working-class backgrounds), as opposed to independence norms (i.e. paving one's path, more commonly valued in middle-class backgrounds), has been shown effective at lessening the achievement gap for first-generation college students by reducing the sense of difficulty perceived by this group, while having no consequences on continuinggeneration students' attendance and performance [9].

Using language that addresses identified cultural norms of specific demographics in fellowship calls and acceptance letters will reduce the identification gap experienced by minorities and increase both engagement and retention of these groups in academia.

Recommendation: We recommend that NASA calls use inclusive language sensitive to the cultural identity and background of traditionally excluded communities. We recommend language that highlights the community aspect of academic work and the support it provides to students over exceptionality and individualistic values, which can be intimidating when it does not reflect individuals' lived experience. Similarly, we recommend the use of language that promotes motivation and interest over previous academic achievements as main selection criteria, and that specifies alternative strategies and resources available to students to meet requirements if necessary.

\section{Summary}

There are short-term and long-term changes to NASA programs, institutional structures, and incentives that have the potential to markedly improve diversity and equal opportunity. We have made ten recommendations that range from easy to implement (1, $7,9,10)$ to those that require research-backed approaches $(5,8)$ and infusion of new resources $(2-4,6)$. We have structured the order of these programs based on a rough estimate of the cumulative positive impact these changes may have for underrepresented groups, but all of these changes positively impact the entire PS\&A community. For example, eliminating bias community-wide through training removes barriers to entry for diverse groups, while also improving the broader community. Infusion of resources into traditionally under represented groups takes dedicated effort, but has the benefit of increasing pipelines of diverse people into the community, which has been shown to improve the quality of work as well [2]. We emphasize that these are meant as starting points for a discussion that must be undertaken as part of a much broader commitment to changing the way the scientific community is built and supported. It will take strong personal and institutional commitments to effect lasting change. 
References: [1a] Hendrix et al. DPS demographics survey: https://dps.aas.org/sites/dps.aas.org/files/reports/survey2020 questionnaire.pdf [1b] DPS survey preliminary results: https://dps.aas.org/sites/dps.aas.org/files/reports/frequencies PRELIMINARY.pdf [1c] Rivera-Valentin et al. Who is Missing in Planetary Science? A demographic study of the planetary science workforce. PS\&A 2020 Decadal white paper. [2] Hayashi, Alden M. Building Better Teams. MIT Sloan Management Review; Cambridge Vol. 45, Iss. 2, (Winter 2004): 5-5. [3] Izenberg, N. "White Papers Cancelled or Downscaled Due to Direct Impact of COVID-19 and National-scale Civil Action", 2020 https://t.co/ZTbd5mhwxB PS\&A 2020 Decadal white paper [4] Singer, K. What are the impacts of performing a Decadal Survey during a global pandemic? https://womeninplanetaryscience.wordpress.com/ [5] Markowitz, Dina G. (2004), Evaluation of the Long-Term Impact of a University High School Summer Science Program on Students' Interest and Perceived Abilities in Science, J. Sci. Ed. Tech. 13, 3. [6] Laursen, Sandra et al. (2007) What Good Is a Scientist in the Classroom? Participation Outcomes and Program Design Features for a Short-Duration Science Outreach Intervention in K-12 Classroom,. Life Sciences Education, 6, 49-64 [7] Clancy, K. B. H., K. M. N. Lee, E. M. Rodgers, and C. Richey (2017), Double jeopardy in astronomy and planetary science: Women of color face greater risks of gendered and racial harassment, J. Geophys. Res. Planets, 122, doi:10.1002/2017JE005256. [8] Carnes, Molly, et al. "Effect of an intervention to break the gender bias habit for faculty at one institution: a cluster randomized, controlled trial." Academic medicine: journal of the Association of American Medical Colleges 90.2 (2015): 221. [9] Stephens, Nicole M., et al. "Unseen disadvantage: how American universities' focus on independence undermines the academic performance of firstgeneration college students." Journal of personality and social psychology 102.6 (2012): 1178. 